\title{
ASYMPTOTICS OF THE SAMPLE COEFFICIENT OF VARIATION AND THE SAMPLE DISPERSION
}

\author{
HANSJÖRG ALBRECHER*, SOPHIE A. LADOUCETTE**, AND JEF L. TEUGELS
}

\begin{abstract}
The coefficient of variation and the dispersion are two examples of widely used measures of variation. We show that their applicability in practice heavily depends on the existence of sufficiently many moments of the underlying distribution. In particular, we offer a set of results that illustrate the behavior of these measures of variation when such a moment condition is not satisfied. Our analysis is based on an auxiliary statistic that is interesting in its own right. Let $\left(X_{i}\right)_{i \geq 1}$ be a sequence of positive independent and identically distributed random variables with distribution function $F$ and define for $n \in \mathbb{N}$

$$
T_{n}:=\frac{X_{1}^{2}+X_{2}^{2}+\ldots+X_{n}^{2}}{\left(X_{1}+X_{2}+\ldots+X_{n}\right)^{2}}
$$

Mainly using the theory of functions of regular variation, we derive weak limit theorems for the properly normalized random quantity $T_{n}$, given that $1-F$ is regularly varying. Following a distributional approach based on $T_{n}$, we then analyze asymptotic properties of the sample coefficient of variation. As a second illustration of the same method, we then turn to the sample dispersion. We also include asymptotic properties of the first moments of these quantities. Finally, we give a distributional result on Student's $t$-statistic which is closely related to $T_{n}$. The main message of this paper is to show that the unconscientious use of some measures of variation can lead to wrong conclusions.
\end{abstract}

\section{IntRoduCtion}

Let $\left(X_{i}\right)_{i \geq 1}$ be a sequence of positive independent and identically distributed random variables with distribution function $F$ and define

$$
T_{n}:=\frac{X_{1}^{2}+X_{2}^{2}+\ldots+X_{n}^{2}}{\left(X_{1}+X_{2}+\ldots+X_{n}\right)^{2}}, \quad n \in \mathbb{N} .
$$

The limiting behavior of arbitrary moments of $T_{n}$ as $n \rightarrow \infty$ has been investigated in [1] under the condition that $X_{1}$ satisfies

$$
1-F(x) \sim x^{-\alpha} \ell(x), \quad x \rightarrow \infty
$$

for some slowly varying function $\ell$ and $\alpha>0$, meaning that $1-F$ is regularly varying with negative index $-\alpha$. It is clear that the smaller the value of $\alpha$, the fatter the tail. Recall that a measurable and ultimately positive function $f$ on $\mathbb{R}_{+}$is regularly varying with index $\gamma \in \mathbb{R}$ (written $f \in \mathrm{RV}_{\gamma}$ ) if $\lim _{x \rightarrow \infty} f(t x) / f(x)=t^{\gamma}$ for all $t>0$. When $\gamma=0$, the function $f$ is said to be slowly varying. We refer to [7] for a textbook treatment on

Date: March 25, 2009.

2000 Mathematics Subject Classification. Primary 62G20; Secondary 60F05.

Key words and phrases. Weak limit theorems; Functions of regular variation; Domain of attraction of a stable law; Sample coefficient of variation; Sample dispersion; Student's $t$-statistic; Extreme value theory.

* Supported by Fellowship F/04/009 of the Catholic University of Leuven and the Austrian Science Foundation Project P18392.

** Supported by the grant BDB-B/04/03 of the Catholic University of Leuven. 
the theory of functions of regular variation.

It is well-known that the tail condition (2) appears as the essential condition in the domain of attraction problem of extreme value theory. For a recent treatment, see [5]. A distribution $F$ satisfying (2) is called of Pareto-type with index $\alpha$. When $0<\alpha<2$, the condition coincides with the additive domain of attraction condition for weak convergence to a non-normal stable law with index $\alpha$.

It is important to remark that distributions satisfying (2) have been used for modeling purposes in numerous practical situations including finance (see e.g. [33, 20, 34]), (re)insurance (e.g. $[22,4,3])$ and geology (see [8]). In telecommunication networks, (2) appears for the modeling of transmission durations and file sizes, see e.g. [29, 30, 47, 48, 39, 40].

In this paper, we focus on weak convergence in deriving limits in distribution for the appropriately normalized random quantity $T_{n}$ under the assumption (2), in that way complementing the results of [1].

For $\beta>0$, define $\mu_{\beta}:=\mathbb{E}\left(X_{1}^{\beta}\right)=\beta \int_{0}^{\infty} x^{\beta-1}(1-F(x)) d x \leq \infty$ and put $\mu:=\mu_{1}$ for convenience. Moreover, we put $\operatorname{Var}\left(X_{1}\right):=\mu_{2}-\mu^{2}$ for the variance of $X_{1}$. Clearly, both the numerator and the denominator in (1) exhibit an erratic behavior if $\mu=\infty$, whereas this is the case only for the numerator if $\mu<\infty$ and $\mu_{2}=\infty$. When $X_{1}$ satisfies the tail condition (2) then $\mu_{\beta}$ is finite if $\beta<\alpha$ but infinite whenever $\beta>\alpha$. In particular, $\mu<\infty$ if $\alpha>1$ while $\mu_{2}<\infty$ as soon as $\alpha>2$. Since the asymptotic behavior of $T_{n}$ is influenced by the finiteness of $\mu_{1}$ and/or $\mu_{2}$, different limiting distributions will consequently show up according to the range of $\alpha$. The possible limit forms are fully covered in our results given in Section 2.

Let $S_{n}:=\sum_{i=1}^{n} X_{i}$ and $V_{n}^{2}:=\sum_{i=1}^{n} X_{i}^{2}$ so that $S_{n} / V_{n}=1 / \sqrt{T_{n}}$. Motivation for our work arose from a number of theorems in connection with weak convergence for the selfnormalized sum $S_{n} / V_{n}$, most prominently those of $[15,32,11,19,18,10,37]$ where the distribution $F$ has support in $\mathbb{R}$ (and not necessarily in $\mathbb{R}_{+}$as supposed here). The line of research leading to these results actually started with the conjecture of [32] stating that $" S_{n} / V_{n}$ is asymptotically normal if [and perhaps only if] $X_{1}$ is in the domain of attraction of the normal law" (and $X_{1}$ is centered). For $X_{1}$ symmetric, the parenthetical "only if" part was proved in [19]. For the general case of a not necessarily symmetric $X_{1}$, the conjecture was proved in [10] sharpening the result of [18] where is was assumed that $S_{n} / V_{n}$ was asymptotically standard normal. Besides, weak convergence results for $S_{n} / V_{n}$ when $X_{1}$ belongs to the domain of attraction of a stable law with index $0<\alpha<2$ have been derived in $[15,32,11,10,37]$. For example, it was shown in [32] that all limit laws of $S_{n} / V_{n}$ for $X_{1}$ in the domain of attraction of a stable law with index $0<\alpha<2$, centered if $1<\alpha<2$ and symmetric if $\alpha=1$, have a subgaussian tail. The asymptotic properties of $T_{n}$ that we derive in Section 2 fit well into the above framework with the additional assumption that we deal with positive random variables.

Armed with the asymptotic results on $T_{n}$, we devote Section 3 to applications on the asymptotic behavior of some widely used measures of variation, namely the sample coefficient of variation and the sample dispersion. Both the coefficient of variation $C V\left(X_{1}\right):=$ $\sqrt{\operatorname{Var}\left(X_{1}\right)} / \mathbb{E} X_{1}$ and the dispersion $D\left(X_{1}\right):=\operatorname{Var}\left(X_{1}\right) / \mathbb{E} X_{1}$ found many applications 

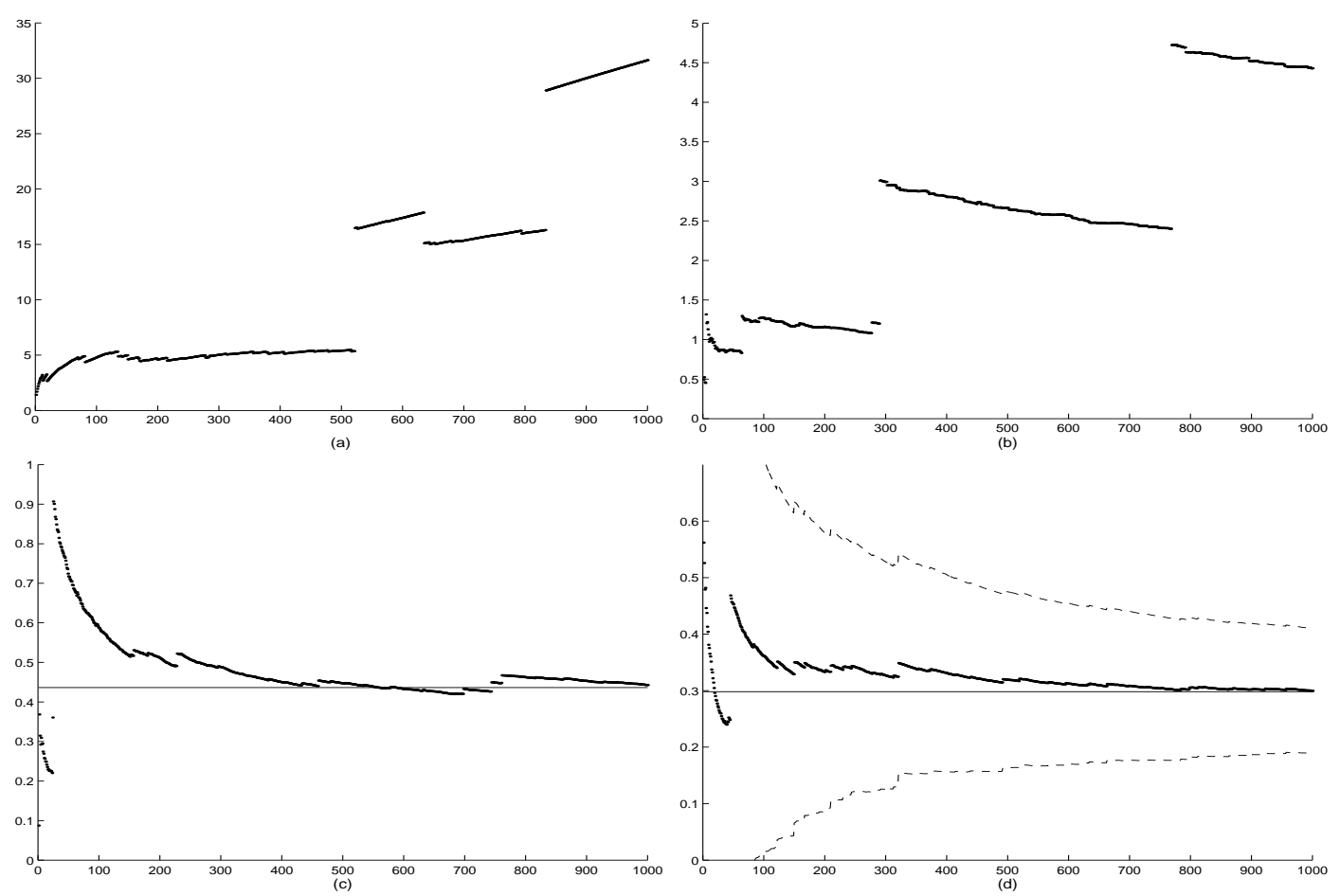

Figure 1. Sample coefficient of variation for $X \sim \operatorname{Par}(\alpha)$ (as a function of $n$ ) with $\alpha=0.5$ (a), 1.5 (b), 3.5 (c), 4.5 (d). In solid lines, true value of $C V(X) \simeq 0.4364$ (c), 0.2981 (d). In dotted lines, $95 \%$-confidence intervals (d).

within the context of insurance and reinsurance, see e.g. [35, 25]. They are usually advocated for comparing variability between distributions with different expected values. As such, they are very popular among actuaries. For an application in actuarial risk theory on mergers of companies, we refer to [17]. We also point out the importance of these quantities in premium calculation in (re)insurance. The reciprocal value $1 / C V\left(X_{1}\right)$ defines the so-called Sharpe's ratio that is very popular in finance as a measure of portfolio performance (see the recent application in [26]). It has been developed as a tool for comparing the risk-adjusted performance of mutual funds, see [42]. We further refer to [41] for an application in the analysis of portfolio selection models with $\alpha$-stable return distributions. We point out that Sharpe's ratio is also used as a measure of market risk, see [36]. Finally, we refer to [13] for a recent illustration of its use in insurance. For a set of papers dealing with the coefficient of variation in the engineering literature, we refer to $[9,31,23,27]$. The coefficient of variation is also used in botany $([45,21])$, in chemistry (see [44]) and in physics (see [16]). Apart from applications in statistics, we mention that the dispersion is often used as a measure of heterogeneity in portfolios.

In practice, the coefficient of variation and the dispersion will be replaced by the corresponding sample values. Let us look at the coefficient of variation first. Since $n T_{n}=$ $\left.\widehat{C V_{n}(X)}\right)^{2}+1$, the quantity $T_{n}$ represents, up to scaling, the sample coefficient of variation $\widehat{C V_{n}(X)}$ of a set of independent observations $X_{1}, \ldots, X_{n}$ from $X$. Since under (2) the existence of moments of $X$ is not always guaranteed, one has to use $\widehat{C V_{n}(X)}$ with 

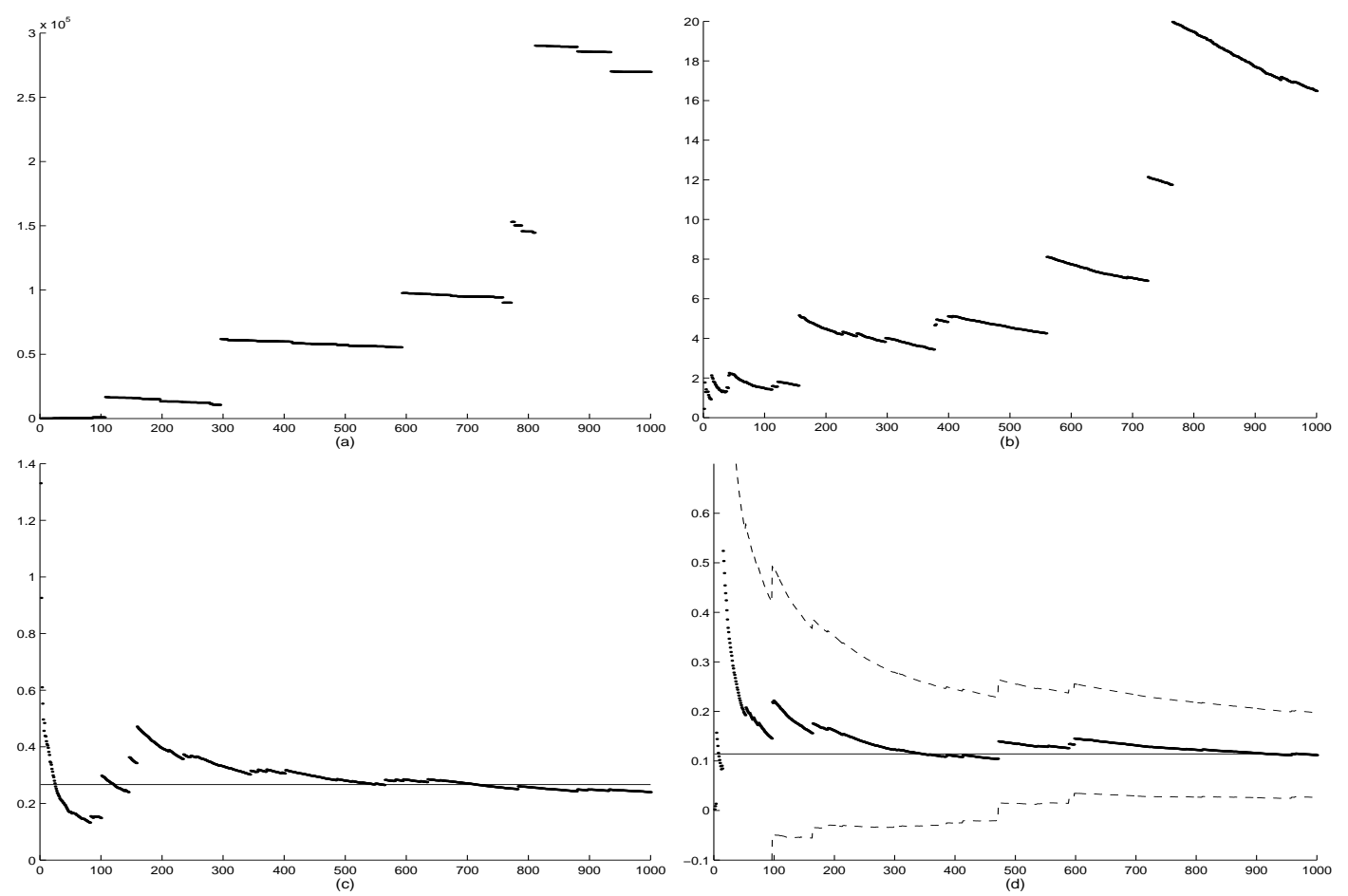

Figure 2. Sample dispersion for $X \sim \operatorname{Par}(\alpha)$ (as a function of $n$ ) with $\alpha=0.5$ (a), 1.5 (b), 3.5 (c), 4.5 (d). In solid lines, true value of $D(X) \simeq$ 0.2667 (c), 0.1143 (d). In dotted lines, 95\%-confidence intervals (d).

great care. We illustrate this with some simulations. To that end, assume that $X$ has a strict-Pareto distribution $\operatorname{Par}(\alpha), \alpha>0$, meaning that $1-F(x)=x^{-\alpha}$ for $x \geq 1$ (note that $(2)$ then holds with $\ell \equiv 1$ ). We depict the behavior of $\left.\widehat{C V_{n}(X}\right)$ in Fig. 1 as a function of the sample size $n$ for $\alpha \in\{0.5,1.5,3.5,4.5\}$. For $\alpha=0.5$ and then $\mu=\infty$ in Fig. 1(a), the jumps in the simulated values of $\widehat{C V_{n}(X)}$ are enormous and indicate a drift to $\infty$. For $\alpha=1.5$, when $\mu<\infty$ but $\mu_{2}=\infty$, Fig. 1(b) shows that the jumps are smaller but the tendency to infinity continues in a similar erratic way. For larger values of $\alpha$, convergence towards the finite value of $C V(X)$ is already visible. When the first two moments exist, the sample coefficient of variation behaves well and converges to the true value, as illustrated in Figs. 1(c)-(d) for $\alpha=3.5$ and $\alpha=4.5$. Only when $\alpha>4$ is it possible to give approximate confidence intervals as illustrated in Fig. 1(d). For these larger values of $\alpha$, it becomes interesting to investigate asymptotic second-order properties.

Very similar remarks apply to the dispersion $D(X)$ and its sample value $\widehat{D_{n}(X)}$. The behavior of $\widehat{D_{n}(X)}$ is illustrated in Fig. 2 as a function of the sample size $n$ for the same values of $\alpha \in\{0.5,1.5,3.5,4.5\}$. Note that for $\alpha=0.5$, the sample values are larger than for the case when $\alpha=1.5$ (see Figs. 2(a)-(b)). Comparing the two sample measures of variation, it is apparent that for a given $\alpha<2$, the speed of divergence to $\infty$ is even faster for $\widehat{D_{n}(X)}$ than for $\widehat{C V_{n}(X)}$. The theoretical results for $\widehat{D_{n}(X)}$ are based on a statistic $C_{n}$ that is similar to $T_{n}$. For this reason, we restrict the theoretical derivations to $T_{n}$. 
The results of Section 2 allow us to derive limits in distribution for the (appropriately normalized) sample coefficient of variation, viewed as a statistic, also in the cases where the first moments do not exist (see Subsection 3.1). In Subsection 3.2, we obtain similar results for the sample dispersion $\widehat{D_{n}(X)}$. Asymptotic properties on moments of the quantities $T_{n}, \widehat{C V_{n}(X)}$ and $\widehat{D_{n}(X)}$ are also specified. Some concluding remarks are given in Section 4. In particular, after pointing out the link between $T_{n}$ and Student's $t$-statistic, we establish a distributional result for the latter quantity.

We end with some notation. Whenever $X_{1}$ satisfies (2) for some $\ell \in \mathrm{RV}_{0}$, we define $\tilde{\ell}(x):=\int^{x}(\ell(t) / t) d t$. It is well-known that $\tilde{\ell} \in \mathrm{RV}_{0}$ again and $\tilde{\ell}(x) / \ell(x) \rightarrow \infty$ for $x \rightarrow \infty$, see e.g. [7]. The symbol $\stackrel{d}{\longrightarrow}, \stackrel{p}{\longrightarrow}, \stackrel{a . s}{\longrightarrow}$ stands for convergence in distribution, in probability and almost surely, respectively. For two positive measurable functions $f$ and $g$, we write $f(x)=O(g(x))$ if $f(x) / g(x) \leq c$ as $x \rightarrow \infty$ for some positive constant $c, f(x)=o(g(x))$ if $f(x) / g(x) \rightarrow 0$ as $x \rightarrow \infty$ and $f(x) \sim g(x)$ if $f(x) / g(x) \rightarrow 1$ as $x \rightarrow \infty$. Finally, $\Gamma(\cdot)$ denotes the gamma function.

\section{Limiting Distributions for $T_{n}$}

We provide limits in distribution for the (normalized) sequence of random variables $\left(T_{n}\right)_{n \geq 1}$, where $F$ satisfies (2). We give a detailed proof for the case $\alpha<1$ (Theorem 2.1) to illustrate the technique, but in order to avoid repetition, we omit the proofs of the cases with $\alpha \geq 1$ as they can be obtained in a similar manner by appropriate scaling. We also would like to point out that an alternative way to derive the limiting results of this section is to use Fourier methods applied to the appropriately centered and normalized two-dimensional sums $\left(\sum_{i=1}^{n} X_{i}, \sum_{i=1}^{n} X_{i}^{2}\right)$ along techniques described in [2] or to use probabilistic methods as those of [11].

We need to distinguish seven cases depending on the value of $\alpha$.

Theorem 2.1. Case 1: $0<\alpha<1$. Then

$$
T_{n} \stackrel{d}{\longrightarrow} \frac{U}{V^{2}}
$$

where the joint distribution of the random variables $(U, V)$ is given by the Laplace transform

$$
\mathbb{E}\left(e^{-s U-t V}\right)=\exp \left\{-2 e^{t^{2} /(4 s)} \int_{0}^{\infty} e^{-\left(u+\frac{t}{2 \sqrt{s}}\right)^{2}}\left(u+\frac{t}{2 \sqrt{s}}\right)\left(\frac{u}{\sqrt{s}}\right)^{-\alpha} d u\right\} .
$$

Proof. Let $1-F(x) \sim x^{-\alpha} \ell(x)$ for some $\ell \in \mathrm{RV}_{0}$ and $0<\alpha<1$. For $\theta>0$ and $\psi \geq 0$, we have

$$
\begin{aligned}
1-\mathbb{E}\left(e^{-\theta X_{1}^{2}-\psi X_{1}}\right) & =\int_{0}^{\infty}\left(1-e^{-\theta x^{2}-\psi x}\right) d F(x) \\
& =\int_{0}^{\infty}(1-F(x)) e^{-\theta x^{2}-\psi x}(2 \theta x+\psi) d x \\
& =2 e^{\psi^{2} /(4 \theta)} \int_{\psi /(2 \sqrt{\theta})}^{\infty} e^{-y^{2}} y\left(1-F\left(\frac{y}{\sqrt{\theta}}-\frac{\psi}{2 \theta}\right)\right) d y
\end{aligned}
$$

where the last equality is obtained by the change of variables $y=\sqrt{\theta} x+\frac{\psi}{2 \sqrt{\theta}}$. 
Define a sequence $\left(a_{n}\right)_{n \geq 1}$ by $1-F\left(a_{n}\right) \sim \frac{1}{n}$, i.e. $\ell\left(a_{n}\right) / a_{n}^{\alpha} \sim \frac{1}{n}$. Now, from the independence of the random variables $X_{i}$ one gets

$$
\begin{aligned}
\mathbb{E}\left(e^{-s \frac{1}{a_{n}^{2}} \sum_{i=1}^{n} X_{i}^{2}-t \frac{1}{a_{n}} \sum_{i=1}^{n} X_{i}}\right) & =\exp \left\{n \log \mathbb{E}\left(e^{-s \frac{1}{a_{n}^{2}} X_{1}^{2}-t \frac{1}{a_{n}} X_{1}}\right)\right\} \\
& \sim \exp \left\{-n\left[1-\mathbb{E}\left(e^{-s \frac{1}{a_{n}^{2}} X_{1}^{2}-t \frac{1}{a_{n}} X_{1}}\right)\right]\right\}
\end{aligned}
$$

and by choosing $\theta=s / a_{n}^{2}$ and $\psi=t / a_{n}$, we obtain from (4) and dominated convergence

$$
\begin{gathered}
\mathbb{E}\left(e^{-\frac{s}{a_{n}^{2}} \sum_{i=1}^{n} X_{i}^{2}-\frac{t}{a_{n}} \sum_{i=1}^{n} X_{i}}\right) \sim \exp \left\{-2 e^{t^{2} /(4 s)} \int_{\frac{t}{2 \sqrt{s}}}^{\infty} y e^{-y^{2}} n\left[1-F\left(a_{n}\left(\frac{y}{\sqrt{s}}-\frac{t}{2 s}\right)\right)\right] d y\right\} \\
\rightarrow \exp \left\{-2 e^{t^{2} /(4 s)} \int_{\frac{t}{2 \sqrt{s}}}^{\infty} y e^{-y^{2}}\left(\frac{y}{\sqrt{s}}-\frac{t}{2 s}\right)^{-\alpha} d y\right\}:=e^{-\psi_{\alpha}(s, t)} .
\end{gathered}
$$

An additional change of variables $y=u+\frac{t}{2 \sqrt{s}}$ leads to

$$
\psi_{\alpha}(s, t)=2 e^{t^{2} /(4 s)} \int_{0}^{\infty} e^{-\left(u+\frac{t}{2 \sqrt{s}}\right)^{2}}\left(u+\frac{t}{2 \sqrt{s}}\right)\left(\frac{u}{\sqrt{s}}\right)^{-\alpha} d u .
$$

Thus, we have shown

$$
\left(\frac{1}{a_{n}^{2}} \sum_{i=1}^{n} X_{i}^{2}, \frac{1}{a_{n}} \sum_{i=1}^{n} X_{i}\right) \stackrel{d}{\longrightarrow}(U, V)
$$

where the joint distribution of $U$ and $V$ is given by (3). The continuous mapping theorem finally gives

$$
T_{n}=\frac{X_{1}^{2}+X_{2}^{2}+\ldots+X_{n}^{2}}{\left(X_{1}+X_{2}+\ldots+X_{n}\right)^{2}} \stackrel{d}{\longrightarrow} \frac{U}{V^{2}}
$$

and the proof is complete.

Remark 2.1. The marginal distribution of $U$ is the weak limit of $\frac{1}{a_{n}^{2}} \sum_{i=1}^{n} X_{i}^{2}$ and hence is determined by taking $t=0$ in (3). This leads to

$$
\mathbb{E}\left(e^{-s U}\right)=\exp \left\{-s^{\alpha / 2} \Gamma(1-\alpha / 2)\right\}
$$

so that $U$ is stable with index $\alpha / 2$. For the marginal distribution of $V$, which is the weak limit of $\frac{1}{a_{n}} \sum_{i=1}^{n} X_{i}$, a little more care is needed, but following the same line of arguments in the proof above with $s=0$, one obtains

$$
\mathbb{E}\left(e^{-t V}\right)=\exp \left\{-t^{\alpha} \Gamma(1-\alpha)\right\}
$$

so $V$ is stable with index $\alpha$, as it should be.

Theorem 2.2. Case 2: $\alpha=1$ and $\mathbb{E}\left(X_{1}\right)=\infty$. Then

$$
\left(\frac{a_{n}^{\prime}}{a_{n}}\right)^{2} T_{n} \stackrel{d}{\longrightarrow} U
$$

where $U$ is a stable random variable with Laplace transform

$$
\mathbb{E}\left(e^{-s U}\right)=\exp \{-\sqrt{\pi s}\}
$$

and where $\left(a_{n}\right)_{n \geq 1}$ is defined by $\ell\left(a_{n}\right) / a_{n} \sim \frac{1}{n}$ and $\left(a_{n}^{\prime}\right)_{n \geq 1}$ is defined by $\tilde{\ell}\left(a_{n}^{\prime}\right) / a_{n}^{\prime} \sim \frac{1}{n}$. 
Remark 2.2. In Theorem 2.2, we have $\left(a_{n}^{\prime} / a_{n}\right)^{2} \in \mathrm{RV}_{0}$ because $a_{n}^{\prime} \in \mathrm{RV}_{1}$ and $a_{n} \in \mathrm{RV}_{1}$. Further, it proves that $a_{n}^{\prime} / a_{n} \rightarrow \infty$ as $n \rightarrow \infty$ since $\tilde{\ell}(x) / \ell(x) \rightarrow \infty$ for $x \rightarrow \infty$.

Theorem 2.3. Case 3: $1<\alpha<2$ (including $\alpha=1$ if $\mathbb{E}\left(X_{1}\right)<\infty$ ). Then

$$
\left(\frac{n}{a_{n}}\right)^{2} T_{n} \stackrel{d}{\longrightarrow} \frac{U}{\mu^{2}}
$$

where $U$ is a stable random variable with Laplace transform

$$
\mathbb{E}\left(e^{-s U}\right)=\exp \left\{-s^{\alpha / 2} \Gamma(1-\alpha / 2)\right\}
$$

and $\left(a_{n}\right)_{n \geq 1}$ is defined by $\ell\left(a_{n}\right) / a_{n}^{\alpha} \sim \frac{1}{n}$.

Remark 2.3. Since $a_{n} \in \mathrm{RV}_{1 / \alpha}$ in Theorem 2.3, we deduce that $\left(n / a_{n}\right)^{2} \in \mathrm{RV}_{2-2 / \alpha}$. When $1<\alpha<2$, we then have $n / a_{n} \rightarrow \infty$ as $n \rightarrow \infty$. When $\alpha=1$ and $\mathbb{E}\left(X_{1}\right)<\infty$, we have $\ell(x)=o(1)$ so that $\left(n / a_{n}\right)^{2} \in \mathrm{RV}_{0}$ satisfies $n / a_{n} \rightarrow \infty$ as $n \rightarrow \infty$.

Theorem 2.4. Case 4: $\alpha=2$ and $\mathbb{E}\left(X_{1}^{2}\right)=\infty$. Then

$$
\left(\frac{n}{a_{n}^{\prime}}\right)^{2} T_{n} \stackrel{p}{\longrightarrow} \frac{2}{\mu^{2}}
$$

where $\left(a_{n}^{\prime}\right)_{n \geq 1}$ is defined by $\tilde{\ell}\left(a_{n}^{\prime}\right) / a_{n}^{\prime 2} \sim \frac{1}{n}$.

Remark 2.4. In Theorem 2.4, since $a_{n}^{\prime} \in \mathrm{RV}_{1 / 2}$, it is clear that $\left(n / a_{n}^{\prime}\right)^{2} \in \mathrm{RV}_{1}$ so that $n / a_{n}^{\prime} \rightarrow \infty$ as $n \rightarrow \infty$.

Remark 2.5. Notice that for all $1 \leq \alpha \leq 2$, the normalizing sequences are regularly varying with index $2-2 / \alpha$ which is increasing with $\alpha$.

In the following results, the case $\alpha>2$ is treated. Since $\mathbb{E}\left(X_{1}\right)<\infty$ and $\mathbb{E}\left(X_{1}^{2}\right)<\infty$, it follows that $n T_{n} \stackrel{a . s .}{\longrightarrow} \frac{\mu_{2}}{\mu^{2}}$ by the law of large numbers. We then deal with second-order weak limit theorems, i.e. we consider convergence in distribution of $k_{n}\left(n T_{n}-\frac{\mu_{2}}{\mu^{2}}\right)$ for appropriate normalizing constants $k_{n} \rightarrow \infty$ as $n \rightarrow \infty$.

Theorem 2.5. Case 5: $2<\alpha<4$ (including $\alpha=2$ if $\mathbb{E}\left(X_{1}^{2}\right)<\infty$ ). Then

$$
\frac{n^{1-2 / \alpha}}{\ell_{1}^{\#}\left(n^{2 / \alpha}\right)}\left(n T_{n}-\frac{\mu_{2}}{\mu^{2}}\right) \stackrel{d}{\longrightarrow} \frac{W}{\mu^{2}}
$$

where $W$ is a stable random variable with index $\alpha / 2$ and $\ell_{1}^{\#} \in \mathrm{RV}_{0}$ is defined as the de Bruyn conjugate of $\ell_{1}(x):=\ell^{-2 / \alpha}(\sqrt{x}) \in \mathrm{RV}_{0}$.

Remark 2.6. In Theorem 2.5, we clearly have $n^{1-2 / \alpha} / \ell_{1}^{\#}\left(n^{2 / \alpha}\right) \in \mathrm{RV}_{1-2 / \alpha}$. In the case $2<\alpha<4$, it follows that the normalizing sequence goes to infinity for $n \rightarrow \infty$. When $\alpha=2$ and $\mathbb{E}\left(X_{1}^{2}\right)<\infty$, it turns out that $\ell(x)=o(1)$ yielding $1 / \ell_{1}^{\#}(n) \rightarrow \infty$ as $n \rightarrow \infty$.

Theorem 2.6. Case 6: $\alpha=4$ and $\mathbb{E}\left(X_{1}^{4}\right)=\infty$. Then

$$
\frac{\sqrt{n}}{\ell_{2}^{\#}(\sqrt{n})}\left(n T_{n}-\frac{\mu_{2}}{\mu^{2}}\right) \stackrel{d}{\longrightarrow} \frac{N(0,1)}{\mu^{2}}
$$

where $N(0,1)$ is a standard normal random variable and $\ell_{2}^{\#} \in \mathrm{RV}_{0}$ is defined as the de Bruyn conjugate of $\ell_{2}(x):=\frac{1}{2 \sqrt{\tilde{\ell}(\sqrt{x})}} \in \mathrm{RV}_{0}$. 
Remark 2.7. In Theorem 2.6, we have $\sqrt{n} / \ell_{2}^{\#}(\sqrt{n}) \rightarrow \infty$ as $n \rightarrow \infty$ since the normalizing sequence is regularly varying with index $1 / 2$. Further, as we have already noticed, the slowly varying function $\ell_{2}^{\#}$ satisfies $\ell_{2}^{\#}(x) \rightarrow \infty$ when $x \rightarrow \infty$.

Finally, if the fourth moment of $X_{1}$ exists then $n T_{n}$ is asymptotically normal (written $\mathrm{AN})$, as given by the following classical result of statistical theory:

Theorem 2.7. Case 7: $\mathbb{E}\left(X_{1}^{4}\right)<\infty$. Then

$$
\sqrt{n}\left(n T_{n}-\frac{\mu_{2}}{\mu^{2}}\right) \stackrel{d}{\longrightarrow} N\left(0, \sigma_{*}^{2}\right)
$$

where

$$
\sigma_{*}^{2}=\frac{\mu_{4}}{\mu^{4}}-\left(\frac{\mu_{2}}{\mu^{2}}\right)^{2}+4\left(\frac{\mu_{2}}{\mu^{2}}\right)^{3}-\frac{4 \mu_{2} \mu_{3}}{\mu^{5}} .
$$

Remark 2.8. In particular, Theorem 2.7 obviously applies if $X_{1}$ is of Pareto-type with index $\alpha>4$ or $\alpha=4$ and $\mathbb{E}\left(X_{1}^{4}\right)<\infty$.

\section{Applications to Risk Measures}

We now look at implications for the asymptotic behavior of two well-known risk measures, namely the sample coefficient of variation (see Subsection 3.1) and the sample dispersion (see Subsection 3.2). More specifically, we derive limit results in terms of distributions and the first moments. In Subsection 3.1, we also include an asymptotic analysis of the first two moments of $T_{n}$, since $T_{n}$ naturally arises in connection with the sample coefficient of variation.

3.1. Asymptotic behavior of the sample coefficient of variation. The coefficient of variation of a positive random variable $X$ is defined by $C V(X):=\frac{\sqrt{\operatorname{Var}(X)}}{\frac{\mathbb{E} X}{X}}$. From a given set of independent observations $X_{1}, \ldots, X_{n}$ of $X$ with sample mean $\frac{\mathbb{E} X}{X}:=n^{-1}\left(X_{1}+\right.$ $\left.\cdots+X_{n}\right)$ and sample variance $S^{2}:=n^{-1} \sum_{i=1}^{n}\left(X_{i}-\bar{X}\right)^{2}, C V(X)$ is estimated by

$$
\widehat{C V_{n}(X)}:=\frac{S}{\bar{X}}
$$

which is called the sample coefficient of variation. Note that there is a slight abuse of notation, since $S$ is usually estimated with normalizing factor $n-1$ instead of $n$, but for the asymptotic considerations in the sequel this becomes irrelevant. The theoretical investigation of properties of the sample coefficient of variation has a long history, see e.g. [43]. The analysis of properties of $\widehat{C V_{n}(X)}$ is typically based on the assumption that sufficiently many moments of $X$ exist, see e.g. $[14,38]$. The special case of normally distributed $X$, where the exact distribution of $\widehat{C V_{n}(X)}$ is available, has received considerable attention in the literature (e.g. $[6,24,46])$. For statistical inference for the coefficient of variation based on small sample size $n$, see [49].

As mentioned before, the existence of moments of $X$ is not always guaranteed in practical applications while there is still a need to study the asymptotic properties of the statistic $\widehat{C V_{n}(X)}$, also in these cases. Due to

$$
n T_{n}=\widehat{C V_{n}(X)}{ }^{2}+1
$$

this can be done by using the results of Section 2. For instance, we have the following. 
(i) $0<\alpha<1$ : If $X$ is in the domain of attraction of a stable law with index $0<\alpha<1$, it follows from Theorem 2.1, Slutsky's theorem and the continuous mapping theorem that $\sqrt{T_{n}-1 / n} \stackrel{d}{\longrightarrow} \sqrt{U} / V$ so that

$$
\frac{\widehat{C V_{n}(X)}}{\sqrt{n}} \stackrel{d}{\longrightarrow} \frac{\sqrt{U}}{V}
$$

where the joint distribution of $U$ and $V$ is given by (3). Thus $\widehat{C V_{n}(X)} \nearrow \infty$ at rate $\sqrt{n}$ as $n \rightarrow \infty$ and the estimator $\widehat{C V_{n}(X)}$ is useless in this case. Note that from Theorem 3.1 of [1], it follows that $\mathbb{E}\left(n T_{n}\right) \sim(1-\alpha) n$ and $\operatorname{Var}\left(n T_{n}\right) \sim \alpha(1-\alpha) n^{2} / 3$.

(ii) $1<\alpha<2$ : If at least the mean exists and $X$ is in the domain of attraction of a stable law with $1<\alpha<2$, Theorem 2.3, Slutsky's theorem and the continuous mapping theorem lead to

$$
\frac{\sqrt{n}}{a_{n}} \widehat{C V_{n}(X)} \stackrel{d}{\longrightarrow} \frac{\sqrt{U}}{\mu}
$$

where the distribution of $U$ is given by $(6)$ and $\left(a_{n}\right)_{n \geq 1}$ is defined by $1-F\left(a_{n}\right) \sim \frac{1}{n}$. This implies $\widehat{C V_{n}(X)} \nearrow \infty$ at rate $a_{n} / \sqrt{n}$ as $n \rightarrow \infty$. Note that $a_{n} / \sqrt{n} \in \mathrm{RV}_{\frac{2-\alpha}{2 \alpha}}$ and then $a_{n} / \sqrt{n} \rightarrow \infty$ as $n \rightarrow \infty$. Theorem 3.3 of [1] shows that $\mathbb{E}\left(n T_{n}\right) \sim \frac{\Gamma(2-\alpha) \Gamma(1+\alpha)}{\mu^{\alpha}} n^{2-\alpha} \ell(n)$ and $\operatorname{Var}\left(n T_{n}\right) \sim \frac{\Gamma(4-\alpha) \Gamma(1+\alpha)}{6 \mu^{\alpha}} n^{3-\alpha} \ell(n)$.

(iii) $2<\alpha<4$ : Here, $X$ is of Pareto-type with index $2<\alpha<4$ and $\sigma^{2}:=\operatorname{Var}(X)<\infty$. From Theorem 2.5, we see that $n T_{n} \stackrel{p}{\longrightarrow} \frac{\mu_{2}}{\mu^{2}}$. Thus, by virtue of the continuous mapping theorem

$$
\widehat{C V_{n}(X)} \stackrel{p}{\longrightarrow} C V(X) .
$$

Moreover, using the identity

$$
b_{n}\left(\widehat{C V_{n}(X)}-C V(X)\right)=\underbrace{\frac{b_{n}\left(n T_{n}-\frac{\mu_{2}}{\mu^{2}}\right)}{2 C V(X)}}_{:=A_{n}}-\underbrace{\frac{b_{n}\left(n T_{n}-\frac{\mu_{2}}{\mu^{2}}\right)^{2}}{2 C V(X)\left(\widehat{C V_{n}(X)}+C V(X)\right)^{2}}}_{:=B_{n}}
$$

one also observes from Theorem 2.5 that for $b_{n}=\frac{n^{1-2 / \alpha}}{\ell_{1}^{\#}\left(n^{2 / \alpha}\right)}$, we have $A_{n} \stackrel{d}{\longrightarrow} \frac{1}{2 \mu \sigma} W$ and $B_{n} \stackrel{d}{\longrightarrow} 0$ leading to

$$
\frac{n^{1-2 / \alpha}}{\ell_{1}^{\#}\left(n^{2 / \alpha}\right)}\left(\widehat{C V_{n}(X)}-C V(X)\right) \stackrel{d}{\longrightarrow} \frac{1}{2 \mu \sigma} W
$$

where $W$ has a stable law with index $\alpha / 2$ and $\ell_{1}^{\#} \in \mathrm{RV}_{0}$ is defined as in Theorem 2.5.

Now, a detailed study of the proof of Theorem 3.4 of [1] gives $\mathbb{E}\left(n T_{n}\right)=\frac{\mu_{2}}{\mu^{2}}+O\left(n^{\max (2-\alpha,-1)}\right)$ which implies (by virtue of the above identity and $B_{n} \geq 0$ ) that $b_{n}\left(\widehat{C V_{n}(X)}-C V(X)\right)$ is uniformly integrable and subsequently $\mathbb{E}\left(\widehat{C V_{n}(X)}\right) \rightarrow C V(X)$ as $n \rightarrow \infty$. Together with (9), it follows that $\widehat{C V_{n}(X)}$ is a consistent and asymptotically unbiased estimator for $C V(X)$. 
From Theorem 3.4 of [1], we also see that $\operatorname{Var}\left(n T_{n}\right) \sim \frac{\Gamma(4-\alpha) \Gamma(1+\alpha)}{6 \mu^{\alpha}} n^{3-\alpha} \ell(n) \nearrow \infty$ for $\alpha<3$ whereas $\operatorname{Var}\left(n T_{n}\right) \searrow 0$ in the case $\alpha \geq 3$ with $\mu_{3}<\infty$. However

$$
\left.\left.\operatorname{Var}\left(\widehat{C V_{n}(X)}\right)=\mathbb{E}\left(\widehat{C V_{n}(X}\right)^{2}\right)-\mathbb{E}^{2}\left(\widehat{C V_{n}(X}\right)\right)=\mathbb{E}\left(n T_{n}-1\right)-\mathbb{E}^{2}\left(\widehat{C V_{n}(X)}\right) \rightarrow 0 .
$$

(iv) $\mu_{4}=\mathbb{E}\left(X^{4}\right)<\infty$ : If the first four moments of $X$ exist then Theorem 2.7 shows that $n T_{n}$ is asymptotically normal. Moreover, the corresponding proof can be repeated using the function $H\left(a_{1}, a_{2}\right)=\sqrt{a_{2}-a_{1}^{2}} / a_{1}$ and this leads to

$$
\sqrt{n}\left(\widehat{C V_{n}(X)}-C V(X)\right) \stackrel{d}{\longrightarrow} N\left(0, \frac{\sigma_{*}^{2} \mu^{2}}{4 \sigma^{2}}\right)
$$

where $\sigma_{*}^{2}$ is given by (8) and $\sigma^{2}:=\operatorname{Var}(X)$. Alternatively, this result can also be obtained by using the decomposition under (iii). The weak law (10) can now be used to set up confidence intervals for the estimation procedure of $C V(X)$.

If $n\left(\widehat{C V_{n}(X)}-C V(X)\right)^{2}$ is uniformly integrable, then one can obtain the limit of $\operatorname{Var}\left(\sqrt{n} \widehat{C V_{n}(X)}\right)$ as the variance of the limiting normal distribution, which by (10) implies that $\operatorname{Var}\left(\widehat{C V_{n}(X)}\right) \sim$ $\sigma_{*}^{2} \mu^{2} /\left(4 \sigma^{2} n\right)$. Thus "the coefficient of variation of the coefficient of variation" behaves like $\left.C V\left(\widehat{C V_{n}(X}\right)\right) \sim \sigma_{*} \mu^{2} /\left(2 \sigma^{2} \sqrt{n}\right)$.

Another approach to determine the limiting behavior of $\left.\mathbb{E}\left(\widehat{C V_{n}(X}\right)^{k}\right)$ based on $k$-statistics can be found in [14].

As a comparison, note that given the uniform integrability of $n\left(n T_{n}-\mu_{2} / \mu^{2}\right)^{2}$, one can obtain the asymptotic behavior of $\operatorname{Var}\left(n T_{n}\right)$ as the corresponding variance of the limiting normal distribution which leads to $\operatorname{Var}\left(n T_{n}\right) \sim \sigma_{*}^{2} / n$. This may also be obtained directly from Theorem 3.4 of [1] by employing a second-order asymptotic analysis.

3.2. Asymptotic behavior of the sample dispersion. Another measure of variation of a positive random variable $X$ that is frequently used in practice is the dispersion defined by $D(X):=\frac{\operatorname{Var}(X)}{\mathbb{E}(X)}$. For instance, in insurance the value of the dispersion allows to determine whether a given portfolio has a Poissonian character or not. With the same notation as before, $D(X)$ is typically estimated by

$$
\widehat{D_{n}(X)}:=\frac{S^{2}}{\bar{X}}
$$

which is called the sample dispersion. Again, the normalizing factor $n$ instead of $n-1$ in $S^{2}$ does not matter asymptotically. If we introduce the quantity

then we have

$$
C_{n}:=\frac{X_{1}^{2}+\cdots+X_{n}^{2}}{X_{1}+\cdots+X_{n}}, \quad n \in \mathbb{N}
$$

$$
\widehat{D_{n}(X)}=C_{n}-\bar{X}
$$

and we can use results from Section 2 to investigate asymptotic properties of the statistic $\widehat{D_{n}(X)}$ also in cases where $X$ is in the domain of attraction of a stable law.

(i) $0<\alpha<1$ : If $X$ is in the domain of attraction of a stable law with index $0<\alpha<1$, it follows from Theorem 2.1 and the continuous mapping theorem that $\frac{1}{a_{n}} C_{n} \stackrel{d}{\longrightarrow} U / V$, 
where $\left(a_{n}\right)_{n \geq 1}$ is defined by $1-F\left(a_{n}\right) \sim \frac{1}{n}$ and the joint distribution of the random variables $(U, V)$ is given by (3). Slutsky's theorem yields

$$
\frac{1}{a_{n}} \widehat{D_{n}(X)} \stackrel{d}{\longrightarrow} \frac{U}{V}
$$

so $\widehat{D_{n}(X)} \nearrow \infty$ at rate $a_{n}$ as $n \rightarrow \infty$ with $a_{n} \in \mathrm{RV}_{1 / \alpha}$. Note that $a_{n} \rightarrow \infty$ for $n \rightarrow \infty$.

Further, it is easy to verify that $\mathbb{E}\left(C_{n} / a_{n}\right)$ and subsequently $\mathbb{E}\left(\widehat{D_{n}(X)}\right)$ tends to $\infty$.

(ii) $1<\alpha<2$ : If at least the mean exists and $X$ is in the domain of attraction of a stable $\overline{\text { law with } 1<\alpha}<2$, Theorem 2.3, Slutsky's theorem and the continuous mapping theorem lead to

$$
\frac{n}{a_{n}^{2}} \widehat{D_{n}(X)} \stackrel{d}{\longrightarrow} \frac{U}{\mu}
$$

where the distribution of $U$ is given by (6) and again $\left(a_{n}\right)_{n \geq 1}$ is defined by $1-F\left(a_{n}\right) \sim \frac{1}{n}$. So, in this case, $\widehat{D_{n}(X)} \nearrow \infty$ at rate $a_{n}^{2} / n$ as $n \rightarrow \infty$ with $a_{n}^{2} / n \in \mathrm{RV}_{\frac{2-\alpha}{\alpha}}$. In particular, $a_{n}^{2} / n \rightarrow \infty$ as $n \rightarrow \infty$. Moreover, along the line of arguments developed in Section 3 of [1] it follows that

$$
\begin{aligned}
\mathbb{E}\left(C_{n}\right) & =n \int_{0}^{\infty} \varphi^{\prime \prime}(s) \varphi^{n-1}(s) d s=\int_{0}^{\infty} \varphi^{\prime \prime}\left(\frac{t}{n}\right) \underbrace{\varphi^{n-1}\left(\frac{t}{n}\right)}_{\rightarrow e^{-\mu t}} d t \\
& \sim \alpha \Gamma(2-\alpha) n^{2-\alpha} \ell(n) \int_{0}^{\infty} t^{\alpha-2} e^{-\mu t} d t=\frac{\alpha \pi}{\sin ((\alpha-1) \pi) \mu^{\alpha-1}} n^{2-\alpha} \ell(n) .
\end{aligned}
$$

Since $\mathbb{E}(\bar{X}) \rightarrow \mu$, this term is negligible and we obtain

$$
\mathbb{E}\left(\widehat{D_{n}(X)}\right) \sim \frac{\alpha \pi}{\sin ((\alpha-1) \pi) \mu^{\alpha-1}} n^{2-\alpha} \ell(n) .
$$

Remark 3.1. From (i) and (ii), we see that the sample dispersion tends to infinity at a faster rate the smaller the index $\alpha$.

(iii) $2<\alpha<4$ : Here, $X$ is of Pareto-type with index $2<\alpha<4$ and we have $\operatorname{Var}(X)<\infty$. Analogous to Theorem 2.5, we obtain for $b_{n}=\frac{n^{1-2 / \alpha}}{\ell_{1}^{\#}\left(n^{2 / \alpha}\right)}$ that

$$
\begin{aligned}
b_{n}\left(\widehat{D_{n}(X)}-D(X)\right) & =b_{n}\left(C_{n}-\frac{\mu_{2}}{\mu}-\bar{X}+\mu\right) \\
& =\underbrace{\frac{b_{n}}{\bar{X}}\left(\frac{1}{n} \sum_{i=1}^{n} X_{i}^{2}-\mu_{2}\right)}_{\stackrel{d}{\longrightarrow} \frac{W}{\mu}}+\underbrace{\frac{\mu_{2} b_{n}}{\bar{X} \mu}(\mu-\bar{X})}_{\stackrel{d}{\longrightarrow} 0}-\underbrace{b_{n}(\bar{X}-\mu)}_{\underline{d} \rightarrow 0}
\end{aligned}
$$

where $W$ has a stable law with index $\alpha / 2$ and $\ell_{1}^{\#} \in \mathrm{RV}_{0}$ is defined as in Theorem 2.5. Slutsky's theorem now leads to

$$
\frac{n^{1-2 / \alpha}}{\ell_{1}^{\#}\left(n^{2 / \alpha}\right)}\left(\widehat{D_{n}(X)}-D(X)\right) \stackrel{d}{\longrightarrow} \frac{W}{\mu} .
$$


Note that the latter implies the following convergence:

$$
\widehat{D_{n}(X)} \stackrel{p}{\longrightarrow} D(X) .
$$

Analogous to Theorem 3.4 of [1], one can derive $\mathbb{E}\left(C_{n}\right)=\frac{\mu_{2}}{\mu}+O\left(n^{\max (2-\alpha,-1)}\right)$ and subsequently $\mathbb{E}\left(\widehat{D_{n}(X)}\right)=D(X)+O\left(n^{\max (2-\alpha,-1)}\right)$. It follows that $\widehat{D_{n}(X)}$ is a consistent and asymptotically unbiased estimator for the dispersion of $X$. Furthermore, by adaptation of the proof of Theorem 3.4 of [1], we derive

$$
\begin{aligned}
\mathbb{E}\left(C_{n}^{2}\right) & =n \int_{0}^{\infty} s \varphi^{(4)}(s) \varphi^{n-1}(s) d s+n(n-1) \int_{0}^{\infty} s\left(\varphi^{\prime \prime}(s)\right)^{2} \varphi^{n-2}(s) d s \\
& \sim \frac{\alpha \Gamma(4-\alpha) \Gamma(\alpha-2)}{\mu^{\alpha-2}} n^{3-\alpha} \ell(n)+\frac{\mu_{2}^{2}}{\mu^{2}}
\end{aligned}
$$

where the first term asymptotically dominates for $\alpha<3$ and the second for $\alpha \geq 3$ with $\mu_{3}<\infty$. Now

$$
\mathbb{E}\left({\widehat{D_{n}(X)}}^{2}\right)=\mathbb{E}\left(C_{n}^{2}\right)-2 \mathbb{E}\left(\frac{1}{n} \sum_{i=1}^{n} X_{i}^{2}\right)+\mathbb{E}\left(\bar{X}^{2}\right) \sim \mathbb{E}\left(C_{n}^{2}\right)-2 \mu_{2}+\mu^{2} .
$$

Thus, $\operatorname{Var}\left(\widehat{D_{n}(X)}\right)=\mathbb{E}\left({\widehat{D_{n}(X)}}^{2}\right)-\mathbb{E}^{2}\left(\widehat{D_{n}(X)}\right)$ is bounded only if the third moment exists and since the constant terms in $\operatorname{Var}\left(\widehat{D_{n}(X)}\right)$ cancel out, we obtain in general

$$
\operatorname{Var}\left(\widehat{D_{n}(X)}\right)=O\left(n^{3-\alpha} \ell(n)\right) \text {. }
$$

(iv) $\mu_{4}=\mathbb{E}\left(X^{4}\right)<\infty$ : If the first four moments of $X$ exist, then using the function $H\left(a_{1}, a_{2}\right)=$ $a_{2} / a_{1}-a_{1}$ in the proof of Theorem 2.7 yields

$$
\sqrt{n}\left(\widehat{D_{n}(X)}-D(X)\right) \stackrel{d}{\longrightarrow} N\left(0, \tilde{\sigma}^{2}\right)
$$

where $\tilde{\sigma}^{2}:=\left(\mu_{2} \mu^{4}-\mu^{6}+\mu_{2}^{3}-2 \mu^{3} \mu_{3}-2 \mu \mu_{2} \mu_{3}+2 \mu_{2}^{2} \mu^{2}+\mu_{4} \mu^{2}\right) / \mu^{4}$. This weak law can be used to set up confidence intervals for the estimation procedure of $D(X)$.

If sufficiently many moments exist then it follows from an adaptation of Theorem 3.4 of [1] that $\mathbb{E}\left(C_{n}^{k}\right)=\left(\frac{\mu_{2}}{\mu}\right)^{k}+O\left(\frac{1}{n}\right)$. In particular, $\operatorname{Var}\left(\widehat{D_{n}(X)}\right)=O\left(\frac{1}{n}\right)$ and, more specifically, from (11) we have $\operatorname{Var}\left(\widehat{D_{n}(X)}\right) \sim \tilde{\sigma}^{2} / n$. Thus "the dispersion of the sample dispersion" behaves like $D\left(\widehat{D_{n}(X)}\right) \sim \tilde{\sigma}^{2} \mu /\left(\sigma^{2} n\right)$.

\section{Concluding Remarks}

As we have illustrated, the practitioner needs to verify statistically the range of $\alpha$-values he might be working with. Fortunately, extreme value theory as in [5] provides a wealth of statistical procedures to acquire sample information on the index. Moreover and as a general statement, one needs to emphasize that also the normalizing sequence $\left(a_{n}\right)_{n \geq 1}$ is unknown in practice and therefore needs to be estimated, see e.g. [28].

The sample dispersion $\widehat{D_{n}(X)}$ is perhaps the most vulnerable among the two statistics that we have considered. In the case $0<\alpha<1, \mathbb{E}\left(\widehat{D_{n}(X)}\right)$ tends to infinity at a rate faster than $a_{n}$. The same applies to the variance whenever $\alpha<2$. Only when $\mathbb{E}\left(X^{4}\right)<\infty$, the estimator for $D(X)$ allows practical confidence intervals. The same applies to the sample coefficient of variation $\widehat{C V_{n}(X)}$ although generally this statistic seems to have slightly 
better properties.

In practice, an alternative approach is to consider statistics like $T_{n}$ and $C_{n}$ directly. Apart from the weak limits treated in this paper, rather explicit results about expectations and variances have been provided in [1].

As a side product, the paper gives the limit distribution of Student's $t$-statistic based upon the sample $X_{1}, \ldots, X_{n}$ when $X_{1}$ is in the domain of attraction of a stable law with index $0<\alpha<1$. Indeed, Student's $t$-statistic $Q_{n}$ is defined by

$$
Q_{n}:=\frac{\sum_{i=1}^{n} X_{i}}{\sqrt{\frac{n}{n-1} \sum_{i=1}^{n}\left(X_{i}-\bar{X}\right)^{2}}}
$$

where $\bar{X}$ is the sample mean. The class of distribution functions on $\mathbb{R}$ such that $Q_{n}$ has a limiting distribution was described in [18]. Also, it was established in $[18,10]$ that $Q_{n}$ is asymptotically (standard) normal if and only if $X_{1}$ is in the domain of attraction of the normal law and $\mathbb{E}\left(X_{1}\right)=0$.

As pointed out by [15], $Q_{n}$ can be written in terms of $T_{n}$ as

$$
Q_{n}=\sqrt{\frac{n-1}{n T_{n}-1}}
$$

so that the limiting distributions for $Q_{n}$ and $1 / \sqrt{T_{n}}$ coincide. When $X_{1}$ belongs to the domain of attraction of a stable law with index $0<\alpha<1$, it then follows from Theorem 2.1 that

$$
Q_{n} \stackrel{d}{\longrightarrow} \frac{V}{\sqrt{U}}
$$

where the joint distribution of $U$ and $V$ is given by (3).

\section{REFERENCES}

[1] H. Albrecher and J.L. Teugels. Asymptotic analysis of a measure of variation. Theory Probab. Math. Stat., 74:1-9, 2006.

[2] A. Araujo, E. Giné. The central limit theorem for real and Banach valued random variables. John Wiley \& Sons, Chichester, 1980.

[3] J. Beirlant, E. Joossens, and J. Segers. Discussion of "Generalized Pareto fit to the society of actuaries' large claims database" by A. Cebrián, M. Denuit and P. Lambert. N. Am. Actuar. J., 8(2):108-111, 2004.

[4] J. Beirlant, G. Matthys, and G. Dierckx. Heavy-tailed distributions and rating. Astin Bull., 31(1):37-58, 2001.

[5] J. Beirlant, Y. Goegebeur, J. Segers, and J.L. Teugels. Statistics of Extremes: Theory and Applications. John Wiley \& Sons, Chichester, 2004.

[6] B. Bennett. On multivariate coefficients of variation. Stat. Hefte, 18(2):123-128, 1977.

[7] N.H. Bingham, C.M. Goldie, and J.L. Teugels. Regular variation. Encyclopedia of Mathematics and its Applications 27. Cambridge University Press, Cambridge, 1987.

[8] J. Caers, J. Beirlant, and M.A. Maes. Statistics for modelling heavy tailed distributions in geology: Part I. Methodology. Math. Geol., 31(4):391-410, 1999.

[9] D.C. Charmpis and G.I. Schueller. Coping with physical uncertainties in structural mechanics: uncertainties modeling, methods of analysis and applications. Technical Report, University of Innsbruck, Austria, 2004.

[10] G.P. Chistyakov and F. Götze. Limit distributions of studentized means. Ann. Probab., 32(1A):28-77, 2004.

[11] M. Csörgö and L. Horváth. Asymptotic representations of self-normalized sums. Probab. Math. Stat., 9(1):15-24, 1988. 
[12] H. Cramér. Mathematical Methods of Statistics. Princeton University Press, 1946.

[13] M.M. Dacorogna and E. Rüttener. Why time-diversified equalisation reserves are something worth having. Converium Research Report, Switzerland, 2006.

[14] F. David. Note on the application of Fisher's k-statistics. Biometrika, 36(3/4):383-393, 1949.

[15] B. Efron. Student's t-test under symmetry conditions. J. Amer. Stat. Assoc., 64:1278-1302, 1969.

[16] J. Feng and D. Brown. Spike output jitter, mean firing time and coefficient of variation. J. Phys. A: Math. Gen., 31(4):1239-1252, 1998.

[17] H.U. Gerber and E.S.W. Shiu. On the merger of two companies. Technical Report, Institut des Sciences Actuarielles, University of Lausanne, Switzerland, 2005.

[18] E. Giné, F. Götze, and D.M. Mason. When is the student $t$-statistic asymptotically standard normal? Ann. Probab., 25(3):1514-1531, 1997.

[19] P.S. Griffin and D.M. Mason. On the asymptotic normality of self-normalized sums. Math. Proc. Camb. Philos. Soc., 109(3):597-610, 1991.

[20] D.M. Guillaume, M.M. Dacorogna, R.R. Davé, U.A. Müller, R.B. Olsen, and O.V. Pictet. From the bird's eye to the microscope: a survey of new stylized facts of the intra-day foreign exchange markets. Finance Stoch., 1(2):95-129, 1997.

[21] M. Hájek and P. Hekera. Can seasonal variation in fen water chemistry influence the reliability of vegetation-environment analyses? Preslia, 76:1-14, 2004.

[22] R.V. Hogg and S.A. Klugman. Loss Distributions. Wiley, New York, 1984.

[23] H.P. Hong and J. Jiang. Ratio between peak inelastic and elastic responses with uncertain structural properties. Can. J. Civ. Eng., 31(4):703-711, 2004.

[24] W. Hürlimann. A uniform approximation to the sampling distribution of the coefficient of variation. Stat. Probab. Lett., 24(3):263-268, 1995.

[25] R. Kaas, M. Goovaerts, J. Dhaene, and M. Denuit. Modern Actuarial Risk Theory. Kluwer Academic Publishers, Boston, 2001.

[26] J. Knight and S. Satchell. A re-examination of Sharpe's ratio for log-normal prices. Appl. Math. Finance, 12(1):87-100, 2005.

[27] K.B. Kordonsky and I. Gertsbakh. Multiple time scales and the lifetime coefficient of variation: engineering applications. Lifetime Data Anal., 3(2):139-156, 1997.

[28] Z. Landsman. Sample quantiles and additive statistics: information, sufficiency, estimation. J. Stat. Plann. Inference, 52(1):93-108, 1996.

[29] W.E. Leland, M.S. Taqqu, W. Willinger, and D.V. Wilson. Statistical analysis of high time-resolution ethernet LAN traffic measurements. In Proceedings of the 25th Symposium on the Interface between Statistics and Computer Science, pages 146-155. Eds. M.E. Tarter, M.D. Lock, 1993.

[30] W.E. Leland, M.S. Taqqu, W. Willinger, and D.V. Wilson. On the self-similar nature of Ethernet traffic (extended version). IEEE/ACM Trans. Networking, 2:1-15, 1994.

[31] J. Li and S.M. Meerkov. On the coefficients of variation of uptime and downtime in manufacturing equipment. Math. Probl. Eng., 2005(1):1-6, 2005.

[32] B.F. Logan, C.L. Mallows, S.O. Rice, and L.A. Shepp. Limit distributions of self-normalized sums. Ann. Probab., 1(5):788-809, 1973.

[33] F.M. Longin. The asymptotic distribution of extreme stock market returns. J. Busin., 69(3):383-408, 1996.

[34] M. Loretan and P.C.B. Phillips. Testing the covariance stationarity of heavy-tailed time series: An overview of the theory with applications to several financial datasets. J. Empi. Finance, 1(2):211-248, 1994.

[35] T. Mack. Schadenversicherungsmathematik. Verlag Versicherungswirtschaft e.v., Karlsruhe, 1997.

[36] C. Marrison. The Fundamentals of Risk Measurement. McGraw-Hill, New York, 2002.

[37] D.M. Mason and J. Zinn. When does a randomly weighted self-normalized sum converge in distribution? Electron. Commun. Probab., 10:70-81, 2005.

[38] Y. Maesono. Mean Squared Errors of Ratio Statistics and Bias Corrections. Research Report SRR03002, Australian National University, 2003.

[39] S. Resnick. Heavy tail modeling and teletraffic data. Ann. Stat., 25(5):1805-1869, 1997.

[40] S. Resnick and H. Rootzén. Self-similar communication models and very heavy tails. Ann. Appl. Probab., 10(3):753-778, 2000.

[41] S.T. Rachev, I. Huber, and S. Ortobelli. Portfolio choice with heavy tailed distributions. Technical Report, University of Karlsruhe, Germany, 2004.

[42] W.F. Sharpe. Mutual fund performance. J. Business, 39(1/2):119-138, 1966. 
[43] R.D. Summers. An inequality for the sample coefficient of variation and an application to variables sampling. Technometrics, 7:67-68, 1965.

[44] C. Seyler, T.B. Hofstetter, and K. Hungerbuhler. Life cycle inventory for thermal treatment of waste solvent from chemical industry: a multi-input allocation model. J. Cleaner Prod., 13(13/14):1211-1224, 2005.

[45] G.P. Svensson, M.O. Hickman, S. Bartram, W. Boland, O. Pellmyr, and R.A. Raguso. Chemistry and geographic variation of floral scent in Yucca filamentosa (Agavaceae). Am. J. Bot., 92(10):1624-1631, 2005.

[46] M. Vangel. Confidence intervals for a normal coefficient of variation. Amer. Stat., 50(1):21-26, 1996.

[47] W. Willinger, M.S. Taqqu, W.E. Leland, and D.V. Wilson. Self-similarity in highspeed packet traffic: analysis and modelling of ethernet traffic measurements. Stat. Sci., 10(1):67-85, 1995.

[48] W. Willinger, M.S. Taqqu, W.E. Leland, and D.V. Wilson. Self-similarity through high variability: statistical analysis of ethernet LAN traffic at the source level (extended version). IEEE/ACM Trans. Networking, 51:71-96, 1997.

[49] A. Wong and J. Wu. Small sample asymptotic inference for the coefficient of variation: normal and nonnormal models. J. Stat. Plann. Inference, 104(1):73-82, 2002.

(H. Albrecher) Institute of Actuarial Science, HeC, University of Lausanne, Quartier UNILDorigny, Batiment Extranef, CH-1015 Lausanne, Switzerland

E-mail address: hansjoerg.albrecher@unil.ch

(S.A. Ladoucette) Department of Mathematics, Catholic University of Leuven, W. de CroyLAAN 54, B-3001 LEUVEN, BElgium

E-mail address: sophie.ladoucette@wis.kuleuven.be

(J.L. Teugels) Department of Mathematics, Catholic University of Leuven, W. de Croylaan 54, B-3001 Leuven, Belgium and EuRAndom, Technical University of Eindhoven, P.O. Box 513, NL-5600 MB Eindhoven, The Netherlands

E-mail address: jef.teugels@wis.kuleuven.be 